\title{
Implementação de um Sistema Inteligente para Previsão do Consumo de Energia Elétrica Residencial
}

\author{
Lucas R. Ilário* Alexandre C. Moreira** \\ Helmo K. Morales-Paredes * \\ * Instituto de Ciência e Tecnologia de Sorocaba - ICTS, Universidade \\ Estadual Paulista - UNESP, Av. Três de março, 511 - Alto da Boa \\ Vista-Sorocaba-SP (e-mail: lucas.ilario@unesp.br, \\ helmo.paredes@unesp.br). \\ ** Núcleo de Inovação, Pesquisa e Ensino em Mecatrônica - NIPEM, \\ Universidade Federal de São João del-Rei, Ouro Branco - MG, \\ (e-mail: amoreira@ufsj.edu.br)
}

\begin{abstract}
The objective of this paper is the application of the Linear Regression technique through the Descendent Gradient method to predict residential electricity consumption. The data used in the analyzes and simulations were obtained through an Intelligent System of Control and Energy Efficiency (SICEE), which provides information on the electricity consumption of a home. The online programming environment Repl.it was the platform used, as well as the programming language Python, for the development of the algorithm. The implemented methodology and the obtained results show the feasibility of its practical application in SICEE.

Resumo: O objetivo deste trabalho é a aplicação da técnica de Regressão Linear por meio do método de Gradiente Descendente para predizer o consumo de energia elétrica residencial. Os dados utilizados nas análises e simulações foram obtidos por meio de um Sistema Inteligente de Controle e Eficiência Energética (SICEE), que afere informações sobre o consumo de energia elétrica de uma residência. O ambiente de programação online Repl.it foi a plataforma utilizada, assim como a linguagem de programação Python, para o desenvolvimento do algoritmo. A metodologia implementada e os resultados obtidos mostram a viabilidade da sua aplicação prática no SICEE.
\end{abstract}

Keywords: Linear Regression; Descendent Gradient; SICEE; Algorithm; Python; Energy; Control; Efficiency; Forecast.

Palavras-chaves: Regressão Linear; Gradiente Descendente; SICEE; Algoritmo; Python; Energia; Controle; Eficiência; Previsão.

\section{INTRODUÇÃO}

A eficiência energética é um tema hodierno e em voga mundialmente, devido a diversos fatores, tal como o aumento da demanda por energia elétrica. Para tanto, a busca por novas fontes de geração de energia elétrica e pelo aperfeiçoamento da eficiência das atuais formas de geração é discutida abertamente entre governos, empresas privadas e comunidades acadêmicas (Banos et al., 2011; Altoé et al., 2017; Mubarok and Septyawan, 2018).

No Brasil, segundo D'alcäntara (2014), os principais programas de eficiência energética são: o Programa Brasileiro de Etiquetagem (PBE), o Programa Nacional de Conservação de Energia Elétrica (PROCEL) e o Programa Nacional de Racionalização do Uso dos Derivados do Petróleo e do Gás Natural (CONPET).

Por outro lado, Tolmasquim (2012) explica que a eficiência energética tem destaque em todos os setores da economia e diz que em 2020 será possível evitar uma demanda de 440 mil barris de petróleo por dia. Penin (2008) argumenta que as campanhas educativas e as ações de eficiência energética auxiliam os consumidores a reduzir o seu consumo com energia elétrica.

Desta forma, prever o consumo do usuário é uma informação importante para o setor elétrico, e consequentemente para auxiliar o planejamento energético, tendo em vista que o excesso ou a falta na geração de energia pode ocasionar perdas econômicas e operacionais (Kaytez et al., 2015; Zipperer et al., 2013).

Dependendo das proporções e condições abordadas, na literatura podem ser encontradas diversas estratégias de se prever o consumo de energia elétrica. Por exemplo, métodos de previsão utilizando diferentes técnicas, tais como: a Lógica Fuzzy (Vasconcelos et al., 2017) e TDNN - Time Delay Neural Network (Rede neural com atraso de tempo) (Souza et al., 2016). Ou ainda, técnicas de redes neurais como o perceptron multicamada e modelos de regressão linear múltiplas para realizar a previsão da demanda anual bruta de eletricidade de um país (Günay, 
2016; He et al., 2012; Zipperer et al., 2013; Kaytez et al., 2015).

Em geral, conhecer o perfil de consumo de energia elétrica possibilita a conscientização para um consumo mais racional, o que ajudaria a desafogar, por exemplo, o Sistema Interligado Nacional (SIN) ou quaisquer outras fontes de energia relacionadas (Altoé et al., 2017).

Segundo Oliveira et al. (2016), na maioria dos países, os edifícios são responsáveis por grande parte da energia consumida, principalmente por conta do desenvolvimento da tecnologia da informação, da internet, e dos dispositivos eletrônicos. Embora a economia resultante da aplicação de programas de eficiência energética no setor residencial seja menor quando comparada com outros setores, por exemplo, o industrial, essa economia não deve ser ignorada, principalmente se formos considerar que no Brasil o setor residencial é o terceiro maior consumidor de energia elétrica.(Altoé et al., 2017).

Com o intuito de nutrir de informações e auxiliar as ações do consumidor que deseja reduzir o seu gasto com energia elétrica mensal, principalmente no atual sistema tarifário de bandeiras vigente no Brasil, onde há variações quanto à tarifação de energia dependendo dos tipos de fontes de geração de energia elétrica. Assim, este trabalho adentra no contexto dos sistemas e ferramentas que auxiliam nessas tomadas de decisões, com o desenvolvimento de um sistema inteligente para prover uma visão geral acerca do consumo de energia elétrica para os consuidores.

Considerando, ainda, as particularidades no desenvolvimento dos dispositivos e algoritmos, e de acordo com os tipos de cargas comumente utilizadas e as faixas de tensão entregues pela concessionária, abordaremos somente a classe de consumidores Residencial (ou grupo B1) neste trabalho.

\section{SISTEMA INTELIGENTE DE CONTROLE E EFICIÊNCIA ENERGÉTICA (SICEE)}

Com o objetivo de auxiliar na tomada de decisão dos consumidores residenciais que viabilize uma redução no consumo de energia elétrica, o SICEE fornece informações detalhadas, sendo um sistema integrado de hardware e software com recursos baseados nos conceitos de Internet das Coisas (Internet of Things - IoT) e sistemas supervisórios.

A arquitetura do SICEE compreende módulos de medição, gateway (arduino + shield ethernet) e servidor multiplataforma, tal como mostrado na Figura 1. O SICEE é capaz de disponibilizar estatísticas detalhadas de consumo por módulo de medição do sistema, identificar o perfil de consumo de energia do usuário, automatizar e bloquear os equipamentos por horários e enviar alertas (mensagens) para dispositivos móveis, além de algumas outras funcionalidades. Os dados armazenados no sistema servem para a elaboração de estatísticas e perfis de consumo.

Entretanto, utilizando técnicas de Machine Learning para tratar e analisar os valores de consumo potência obtidos pelos módulos de medição do SICEE, desenvolveu-se um algoritmo para realizar a previsão do consumo de energia elétrica, de modo que o usuário possa ter conhecimento, de

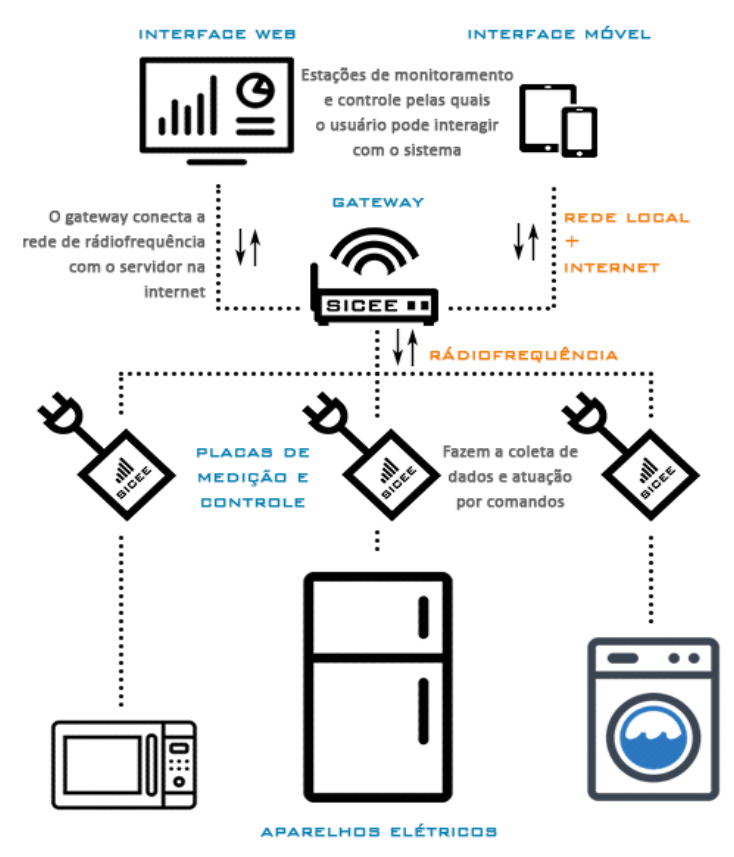

Figura 1. Arquitetura do SICEE.

maneira aproximada, do quanto ele precisará desembolsar ao final do mês de acordo com o seu perfil de consumo.

Considerando a característica acumulativa e a correlação linear entre os dados medidos (Figura 2) e o perfil do consumidor residencial, a técnica escolhida para previsão foi a de Regressão Linear.

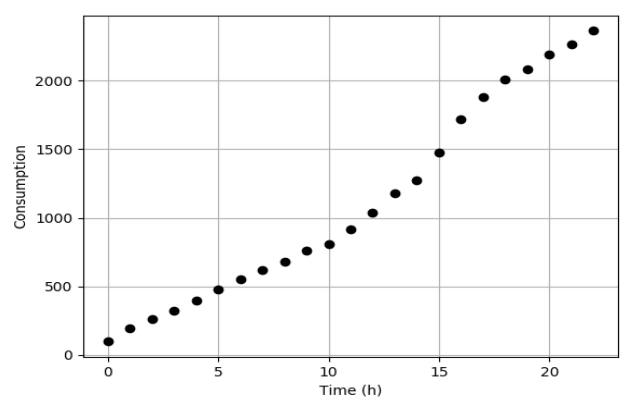

Figura 2. Consumo total acumulado do microondas, da geladeira e da máquina de lavar.

\section{TÉCNICA DE REGRESSÃO LINEAR}

Segundo Santos (2018), a técnica de regressão linear permite estimar relações entre variáveis, além de formular modelos estatísticos, e é utilizada, na maioria dos casos, para modelos de previsão.

A técnica de Regressão Linear Simples é baseada num modelo com uma única variável dependente " $h$ " relacionada a uma variável independente "t", além de depender da forma de distribuição dos dados a serem expressos pela linha de regressão (Kavitha et al., 2016; Santos, 2018; Wang and Yong, 2008). De acordo com Song et al. (2016), Vasconcelos et al. (2017), e Silva (2017), "a" e "b" são os coeficientes de regressão estimados e "h" é o valor predito. Denomina-se essa função "h" de Hipótese, tal que: 


$$
h(t)=a+b * t
$$

Para tanto, precisa-se ajustar a função linear aos dados conhecidos, modelando o problema a fim de minimizar uma função Custo J, onde " $x$ " são os valores reais conhecidos e "m" é o número de iterações ocorridas para se chegar a melhor solução.

$$
J(a, b)=\frac{1}{2 m} \sum_{i=1}^{m}\left(x^{i}-h\left(t^{i}\right)\right)^{2}
$$

E para o cálculo do ponto de mínimo, emprega-se o método do Gradiente Descendente, buscando otimizar a função Custo associada a função Hipótese, tal que a eq. (3) é a função Custo derivada em relação a "a", e a eq. (4) é a função Custo derivada em relação a "b".

$$
\begin{gathered}
\frac{d}{d_{a}} J(a, b)=\frac{1}{m} \sum_{i=1}^{m}-2 \cdot\left(x^{i}-h^{i}\right) \\
\frac{d}{d_{b}} J(a, b)=\frac{1}{m} \sum_{i=1}^{m}-2 \cdot t^{i} \cdot\left(x^{i}-h^{i}\right)
\end{gathered}
$$

Essas funções são necessárias para a obtenção dos novos valores dos coeficientes de regressão "a" e "b", que são atualizados simultaneamente subtraindo deles as respectivas derivadas parciais da função Custo (Silva, 2017), onde " $\alpha$ " é a taxa de aprendizado, tal que:

$$
\begin{gathered}
a_{\text {novo }}=a_{\text {velho }}-\alpha \cdot \frac{d}{d_{a}} J(a, b) \\
b_{\text {novo }}=b_{\text {velho }}-\alpha \cdot \frac{d}{d_{b}} J(a, b)
\end{gathered}
$$

Quando se encontra o ponto de mínimo, a nova função $\mathrm{h}(\mathrm{t})$ é a mais próxima dos dados utilizados, além de engendrar a possibilidade de previsão de dados futuros (De Cola and Mongelli, 2018; Yang et al., 2019).

\section{METODOLOGIA PROPOSTA}

Com o Sistema Inteligente de Controle e Eficiência Energética (SICEE) desenvolvido, iniciou-se o engendramento de um algoritmo de previsão de consumo de energia. Segundo Mubarok and Septyawan (2018), qualquer técnica de previsão necessita atender as características de precisão, custo e conveniência, tais como as de Redes Neurais e Lógica Fuzzy, mas optou-se pela implementação da técnica de Regressão Linear a fim de analisar suas possibilidades e perspectivas para aferir valores futuros do consumo de energia elétrica.

Para a implementação do algoritmo de previsão do consumo de energia no SICEE, serão utilizados os valores obtidos pelos módulos de medição conforme a Figura 3.

A implementação dos módulos de medição do SICEE são mostrados na Figura 4. Os mesmos realizaram a aferição dos valores de consumo da Geladeira, do Microondas e da Máquina de lavar roupas, considerando toda a potência

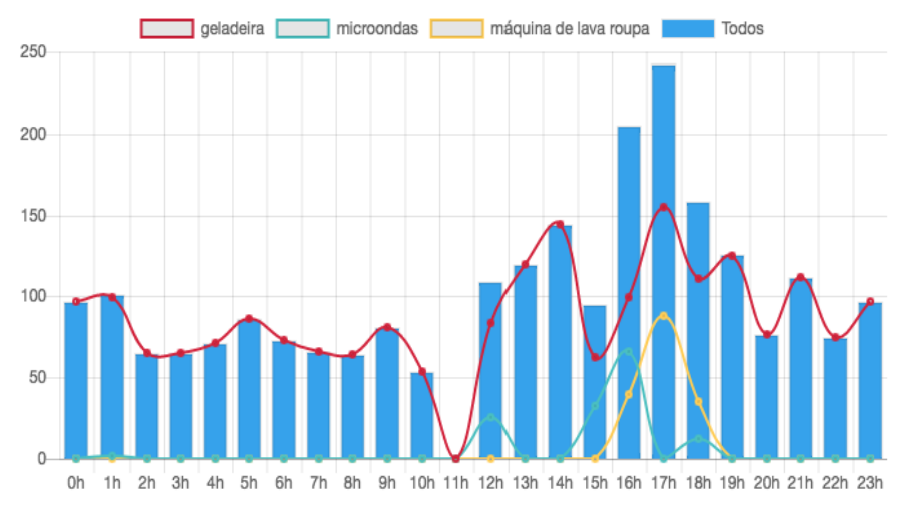

Figura 3. Consumo por hora do dia.

usada por esses aparelhos a cada hora durante o período de um dia, conforme apresentado na Figura 3. Além disso, na Tabela 1, são apresentados o consumo individual de cada aparelho, assim como os valores do Consumo Total Acumulado dos três aparelhos que foram utilizados para efetuar a Regressão Linear.

Tabela 1. Consumo de Dispositivos.

\begin{tabular}{|l|l|l|c|r|}
\hline $\begin{array}{l}\text { Hora do } \\
\text { dia }(\mathrm{h})\end{array}$ & $\begin{array}{l}\text { Gelade- } \\
\text { ira }(\mathrm{W})\end{array}$ & $\begin{array}{l}\text { Micro- } \\
\text { ondas }(\mathrm{W})\end{array}$ & $\begin{array}{c}\text { Máq. de } \\
\text { lavar }(\mathrm{W})\end{array}$ & $\begin{array}{r}\text { Total } \\
(\mathrm{W})\end{array}$ \\
\hline 1 & 97 & 0 & 0 & 97 \\
\hline 2 & 100 & 0 & 0 & 197 \\
\hline 3 & 63 & 0 & 0 & 260 \\
\hline 4 & 64 & 0 & 0 & 324 \\
\hline 5 & 70 & 0 & 0 & 394 \\
\hline 6 & 86 & 0 & 0 & 480 \\
\hline 7 & 72 & 0 & 0 & 552 \\
\hline 8 & 64 & 0 & 0 & 616 \\
\hline 9 & 63 & 0 & 0 & 679 \\
\hline 10 & 80 & 0 & 0 & 759 \\
\hline 11 & 52 & 0 & 0 & 811 \\
\hline 12 & 82 & 23 & 0 & 916 \\
\hline 13 & 119 & 0 & 0 & 1035 \\
\hline 14 & 145 & 0 & 0 & 1180 \\
\hline 15 & 62 & 31 & 39 & 1478 \\
\hline 16 & 100 & 6 & 88 & 1720 \\
\hline 17 & 154 & 0 & 37 & 1881 \\
\hline 18 & 111 & 13 & 0 & 2006 \\
\hline 19 & 125 & 0 & 0 & 2082 \\
\hline 20 & 76 & 0 & 0 & 2194 \\
\hline 21 & 112 & 0 & 0 & 2268 \\
\hline 22 & 74 & 0 & 0 & 04 \\
\hline 23 & 96 & 0 & 0 & \\
\hline
\end{tabular}

Partindo dessas informações e utilizando a linguagem de programação Python, portanto, o algoritmo desenvolvido visa encontrar a melhor função para um conjunto de dados já conhecidos. Ademais, como o objetivo é estimar o montante consumido no final do mês, os dados utilizados encontrados para a Regressão Linear foram os valores referentes ao Consumo Total Acumulado a cada hora, conforme mostrado na quinta coluna da Tabela 1.

\section{RESULTADOS E DISCUSSÕES}

O algoritmo de Regressão Linear é inicializado com coeficientes de regressão "a" e "b" estimados a partir dos dados conhecidos (97 e 150, respectivamente), que correspondem aos dados da primeira amostra do Consumo Total Acumulado. 


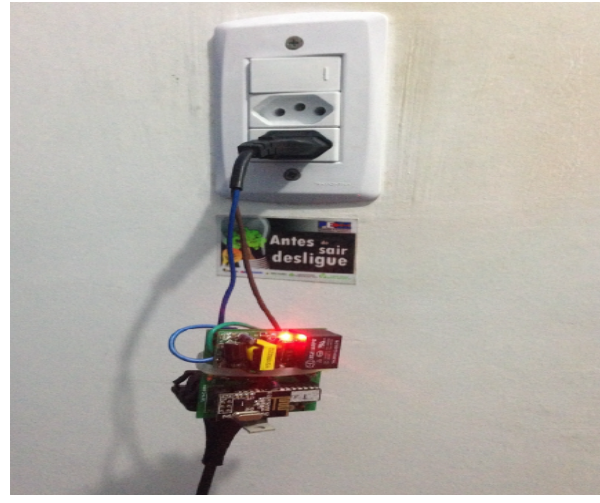

(a) Módulo medidor ligado.

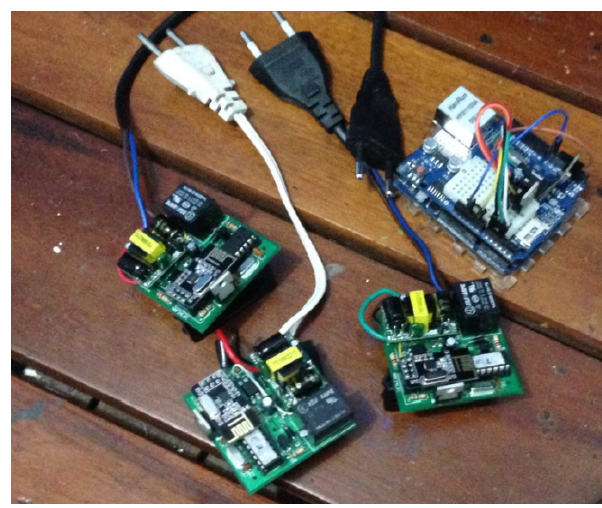

(b) módulos medidores usados na aferição dos dados.

Figura 4. Módulos medidores do SICEE.

Além disso, variando o " $\alpha$ ", obteve-se os valores apresentados na Tabela 2. Percebe-se que com a mudança de " $\alpha$ ", a função Custo $h(t)$ permaneceu praticamente a mesma. No entanto, os outros valores tiveram mudanças significativas, onde um menor valor de " $\alpha$ " resulta numa maior precisão, como observado nas Figuras 5 e 6.

Tabela 2. Resultados dos testes.

\begin{tabular}{|l|c|r|}
\hline Variáveis & Dados do $1^{\circ}$ teste & Dados do $2^{\circ}$ teste \\
\hline $\mathrm{a}$ & 97 & 97 \\
\hline $\mathrm{b}$ & 150 & 150 \\
\hline$\alpha$ & 0,001 & 0,0001 \\
\hline $\mathrm{h}(\mathrm{t})$ & 2363.87 & 2363,96 \\
\hline $\mathrm{J}(\mathrm{a}, \mathrm{b})$ & 23342.81 & 8192.22 \\
\hline$\frac{d}{d_{a}} J(a, b)$ & 334.49 & 36.44 \\
\hline$\frac{d}{d_{b}} J(a, b)$ & -5.69 & -1.58 \\
\hline $\mathrm{a}_{\text {novo }}$ & 95,11 & -216.59 \\
\hline $\mathrm{b}_{\text {novo }}$ & 98,64 & 112.19 \\
\hline iterações & 2 & 23213 \\
\hline
\end{tabular}

Para uma melhor visualização dos valores e da função Hipótese, as Figuras 5 e 6(a) apresentam os dados para 48 horas (até o fim do dia seguinte). Observa-se que a função Hipótese da Figura 6(a) apresenta uma melhor estimação da função Hipótese do que a da Figura 5, porém requer um esforço computacional maior, em função de uma taxa de aprendizado menor. Por outro lado, a previsão para 720 horas pode ser observada na figura $6(\mathrm{~b})$.

Com base nos resultados obtidos, confirma-se que a técnica de Regressão Linear pode ser utilizada para realizar a previsão do consumo de energia elétrica.
Ademais, frisa-se que com o tempo, e o incremento dos dados armazenados, a precisão da função Hipótese se mantém conforme os conceitos da técnica de Regressão Linear.

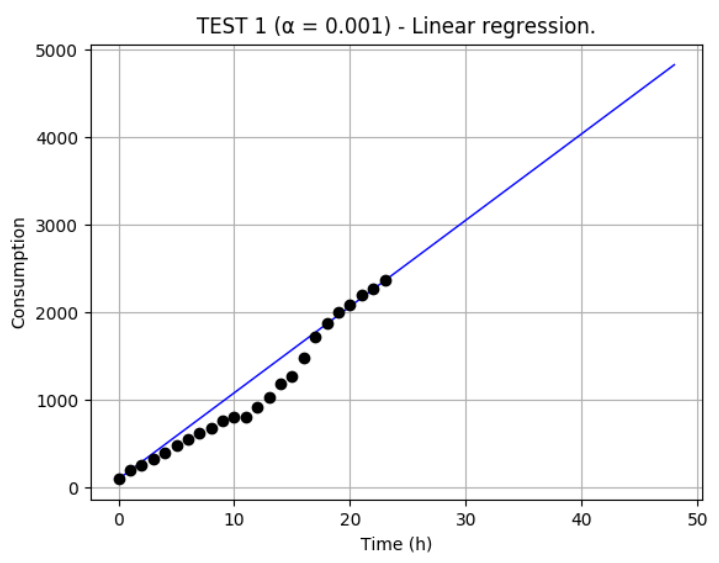

Figura $5.1^{\circ}$ teste (Previsão para $48 \mathrm{~h}$ )

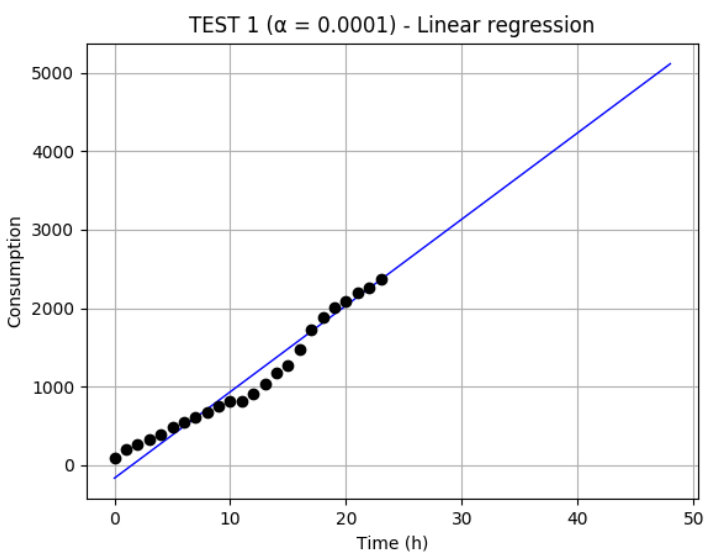

(a) Previsão para 48h.

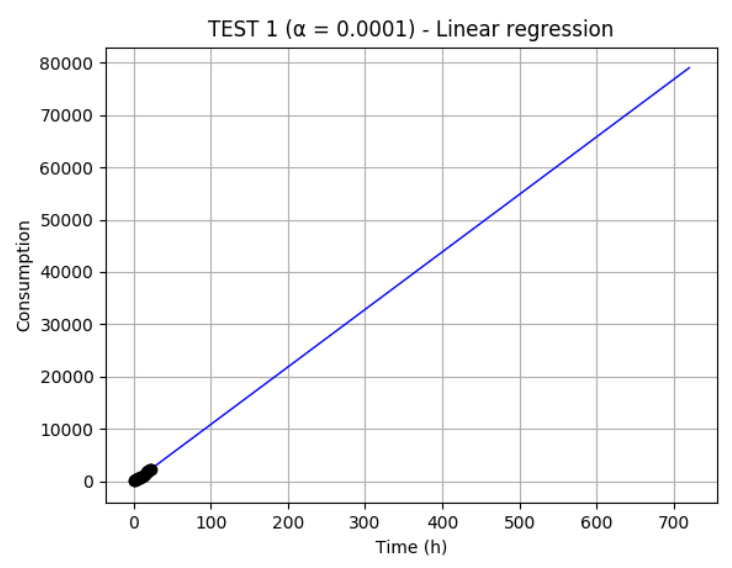

(b) Previsão para $720 \mathrm{~h}$.

Figura 6. $2^{\mathrm{o}}$ teste.

\section{CONCLUSÕES}

Dentre as várias possibilidades de métodos e técnicas para engendrar a previsão do consumo residencial de eletri- 
cidade, o método de Regressão Linear Simples apresentou resultados satisfatórios, atendendo as características apontadas por Mubarok and Septyawan (2018) trazendo uma função de previsão aproximada ao esperado, um custo baixo e uma grande conveniência. Ademais, espera-se que em trabalhos futuros seja possível verificar a viabilidade da aplicação da Regressão Linear Múltipla, e também discutir as particularidades da abordagem proposta neste trabalho e as vantagens e desvantagens perante outras metodologias encontradas na literatura.

\section{AGRADECIMENTOS}

Os autores agradecem o suporte financeiro do $\mathrm{CNPq}$ (Processos 431944/2018-0 e 311332/2018-8) e da FAPESP (Processo 2016/08645-9 e 2018/20708-1) no desenvolvimento desta pesquisa.

\section{REFERÊNCIAS}

Altoé, L., Costa, J.M., Oliveira Filho, D., Martinez, F.J.R., Ferrarez, A.H., and Viana, L.d.A. (2017). Políticas públicas de incentivo à eficiência energética. Estudos Avançados, 31(89), 285-297.

Banos, R., Manzano-Agugliaro, F., Montoya, F., Gil, C., Alcayde, A., and Gómez, J. (2011). Optimization methods applied to renewable and sustainable energy: A review. Renewable and sustainable energy reviews, 15(4), 1753-1766.

D'alcäntara, A.J.R. (2014). Análise do programa brasileiro de etiquetagem energética predial. $100 \mathrm{f}$.

De Cola, T. and Mongelli, M. (2018). Adaptive time window linear regression for outage prediction in $\mathrm{q} / \mathrm{v}$ band satellite systems. IEEE Wireless Communications Letters, 7(5), 808-811.

Günay, M. (2016). Forecasting annual gross electricity demand by artificial neural networks using predicted values of socio-economic indicators and climatic conditions: Case of turkey. Energy Policy, 90, 92-101.

He, Y., Li, Y., and Zhao, X. (2012). Analysis of energy saving potential of power industry in china based on multiple liner regression model. In 2012 8th International Conference on Computing Technology and Information Management (NCM and ICNIT), volume 1, 206-209. IEEE.

Kavitha, S., Varuna, S., and Ramya, R. (2016). A comparative analysis on linear regression and support vector regression. In 2016 Online International Conference on Green Engineering and Technologies (IC-GET), 15. IEEE.

Kaytez, F., Taplamacioglu, M.C., Cam, E., and Hardalac, F. (2015). Forecasting electricity consumption: A comparison of regression analysis, neural networks and least squares support vector machines. International Journal of Electrical Power \& Energy Systems, 67, 431-438.

Mubarok, H. and Septyawan, R. (2018). Analysis of pln's electrical energy demand in the area of batamindonesia using the linear regression method. In 2018 4 th International Conference on Science and Technology (ICST), 1-5. IEEE.

Oliveira, L.K.S., Rêgo, R.M., Frutuoso, M.N.M.A., and Rodrigues, S.S.F.B. (2016). Simulação computacional da eficiência energética para uma arquitetura sustentável. $H O L O S, 4,217-230$.
Penin, C. (2008). Combate, prevenção e otimização das perdas comerciais de energia elétrica. $214 f$. Ph.D. thesis, Tese (Doutorado)-Curso de Engenharia, Universidade de São Paulo, São Paulo.

Santos, D.S. (2018). Aprendizado de máquina: estatística bayesiana em método de regressão linear simples com aplicação em magnitudes de quasares. Monografia (Bacharelado em Física), UFRGS (Instituto de Física, Universidade Federal do Rio Grande do Sul), Rio Grande do Sul, Brasil.

Silva, J.P.B.C. (2017). Modelos de Regressão Linear e Logística utilizando o software $R$. Ph.D. thesis.

Song, S., Ge, L., and Zhang, Z. (2016). Accurate position estimation of srm based on optimal interval selection and linear regression analysis. IEEE Transactions on Industrial Electronics, 63(6), 3467-3478.

Souza, W.A. et al. (2016). Estudos de técnicas de análise e tecnologias para o desenvolvimento de medidores inteligentes de energia residenciais.

Tolmasquim, M.T. (2012). Perspectivas e planejamento do setor energético no brasil. Estudos avançados, 26(74), 247-260.

Vasconcelos, L.P., Viterbo Filho, J., Vermelha, C.P., Niterói, R., Coelho, I.M., and da Silva, J.M.M. (2017). Uma comparação de técnicas de regressão para a previsão de consumo de energia residencial no cenário nacional. In XLIX Simpósio Brasileiro de Pesquisa Operacional.

Wang, Z.Z. and Yong, J.H. (2008). Texture analysis and classification with linear regression model based on wavelet transform. IEEE transactions on image processing, 17(8), 1421-1430.

Yang, X., Yang, H., Zhang, F., Zhang, L., Fan, X., Ye, Q., and $\mathrm{Fu}$, L. (2019). Piecewise linear regression based on plane clustering. IEEE Access, 7, 29845-29855.

Zipperer, A., Aloise-Young, P.A., Suryanarayanan, S., Roche, R., Earle, L., Christensen, D., Bauleo, P., and Zimmerle, D. (2013). Electric energy management in the smart home: Perspectives on enabling technologies and consumer behavior. Proceedings of the IEEE, 101(11), 2397-2408. 